\title{
On Some Global Solution of the Basic Equations in the Geodesic Mappings' Theory of Riemannian Spaces
}

\author{
E. N. Sinyukova ${ }^{1, *}$, O. L. Chepok $^{2}$ \\ ${ }^{1}$ Department of Mathematics and Statistics, South Ukrainian National Pedagogical University named after K. D. Ushynsky, Odessa, \\ 65026, Ukraine \\ ${ }^{2}$ Department of Physics, South Ukrainian National Pedagogical University named after K. D. Ushynsky, Odessa, 65026, Ukraine
}

Received August 10, 2020; Revised November 6, 2020; Accepted November 29, 2020

\section{Cite This Paper in the following Citation Styles}

(a): [1] E. N. Sinyukova, O. L. Chepok, "On Some Global Solution of the Basic Equations in the Geodesic Mappings' Theory of Riemannian Spaces," Mathematics and Statistics, Vol. 8, No. 6, pp. 693 - 698, 2020. DOI: 10.13189/ms.2020.080609.

(b): E. N. Sinyukova, O. L. Chepok (2020). On Some Global Solution of the Basic Equations in the Geodesic Mappings' Theory of Riemannian Spaces. Mathematics and Statistics, 8(6), 693 - 698. DOI: 10.13189/ms.2020.080609.

Copyright $\bigcirc 2020$ by authors, all rights reserved. Authors agree that this article remains permanently open access under the terms of the Creative Commons Attribution License 4.0 International License

\begin{abstract}
It is well known that concepts of a geodesic line and a geodesic mapping are among the most fundamental concepts of classical theory of Riemannian spaces. In geometry, concept of Riemannian space has been formed as a generalization of the concept of a smooth surface in a three-dimensional Euclidean space. It has turned out to be possible to extend to Riemannian space the concept of a geodesic point of a curve and to represent a geodesic line of Riemannian space as a curve that consists exclusively of geodesic points. The fact has allowed understanding not only the local but also the global character of basic equations of geodesic mappings' theory of Riemannian spaces that have been originally received as a result of local investigations. An example of the global solution of the so-called new form of basic equations in the theory of geodesic mappings of Riemannian spaces is built in the article. Sphere $S^{n}$ that is considered as a subset of Euclidean space $E^{n+}, n \in N$, forms its topological background. Investigations are based on the concept of equidistant Riemannian space. They are carried out according to the atlas that consists of two charts, obtained with the help of a stereographic projection.
\end{abstract}

Keywords Riemannian Space, Geodesic Line, Local Geodesic Mapping, Global Geodesic Mapping, Equidistant Riemannian Space, Sphere

\section{Introduction}

We will consider as a differentiable manifold $M^{n}$ of the class $C^{r}, n, r \in N, r>1$, a Hausdorff topological space that satisfies the second axiom of countability, every point of which has a neighborhood that is homeomorphic to some domain of the space $R^{n}$. The homeomorphism establishes in the every of such neighborhoods the so-called local coordinate system that turns it into the coordinate neighborhood. Any two coordinate neighborhoods are $C^{\prime \prime}$-related in the sense that appeared in the case of their nonempty intersection functions of transformation of the one coordinate system to the other, are the smooth functions of the class $C^{r}$. By the space $R^{n}$ we will understand an arithmetic Euclidean space of ordered n-tuples of real numbers, endowed by topology induced by its natural metric ([1], for example). As it is known ([2], for example) on such a manifold there exists, not only locally, but also on the whole, a smooth of the class $C^{r-1}$ twice covariant metric tensor (that can be established by infinite means, that not necessary represents a positive defined quadratic form in the every point of $M^{n}$ ) that turns the manifold into Riemannian $C^{r}$-space $V^{n}$.

One-dimensional, smooth of the class $C^{k}, k \leq r$, submanifold $L$ of some manifold $M^{n}$ is called a smooth of the class $C^{k}$ simple curve of the manifold. The possibility of coordinate neighborhoods of a manifold to be divided unlimited onto the smallest ones allows to secure the existence of such a coordinate neighborhood 
for the every point of the simple $C^{k}$-curve $L$ of the manifold $M^{n}$ according to which the curve $L$ has a coordinate representation of the type

$$
\mathrm{x}^{\mathrm{h}}=\mathrm{x}^{\mathrm{h}}(\mathrm{t}), \mathrm{t} \in \mathrm{I}, \quad \mathrm{x}^{\mathrm{h}}(\mathrm{t}) \in C^{k}, \quad \mathrm{~h}=\overline{1, \mathrm{n}},
$$

where $I$ is some non-empty interval of the coordinate line.

A point $P$ of the simple $C^{2}$-curve $L$ of Riemannian $C^{r}$-space $V^{n}(n, r \in N, r \geq 2)$ is called a geodesic point of the curve if in this point the vector

$$
\eta^{h}(t)=\frac{d x^{h}}{d t}
$$

tangent to the $L$, satisfies the conditions

$$
\eta_{, \alpha}^{h} \eta^{\alpha} \equiv \frac{d \eta^{h}}{d t}+\Gamma_{\alpha \beta}^{h} \eta^{\alpha} \eta^{\beta}=\rho \eta^{h}
$$

where the invariant $\rho$ depends only on $t$. It is clear that conditions (1) represent restrictions to the meanings at the point $P$ of the components of the corresponding geometrical objects of the considered Riemannian space $V^{n}$ according to some coordinate neighborhood of the point $P$. In particular, $\Gamma_{\alpha \beta}^{h}(h, \alpha, \beta=\overline{1, n})$ are the meanings of components of the object of affine connection at the point $P$. The geometrical sense of conditions (1) that means their independence from the chosen in the neighborhood of the point $P$ permissible for the considered Riemannian space local coordinate system, is determined by their tensor character.

If $C^{2}$-curve $L$ of Riemannian $C^{r}$-space $V^{n} \quad(n, r \in N, r$ $\geq 2$ ) consists only of geodesical points, it is called a geodesic line of the space [3]. It is proved that for any Riemannian space $V^{n}$ of the class $C^{r}, r>2$, though a given point $P$ in any given direction $\eta^{h}$ there passes a unique geodesic line (see, for example, [4]).

If there exists a $C^{r}$-diffeomorphic mapping of Riemannian $C^{r}$-space $V^{n}$ onto Riemannian $C^{r}$-space $\bar{V}^{n}$, $n, r \in N, r>1$, according to which every geodesic line of the space $V^{n}$ turns into a geodesic line of the space $\bar{V}^{n}$ then, by the definition, the mapping is called a geodesic mapping "on the whole" of the Riemannian space $V^{n}$ onto the Riemannian space $\bar{V}^{n}$.

And if some mapping $f$, defined in some neighborhood $U$ of the point $P$ of Riemannian $C^{r}$-space $V^{n}, n, r \in N$, $r>1$, as a $C^{\prime \prime}$-diffeomorphism that preserves geodesic lines, maps the neighborhood onto some neighborhood $\bar{U}$ of some $C^{r}$-space $\bar{V}^{n}$, it is called a geodesic mapping directly of the neighborhood $U$ of the point $P$. If such mappings can be defined for some neighborhoods of every point of the space $V^{n}$,we say that the space $V^{n}$ locally admits geodesic mappings.

It is clear that any geodesic mapping for the space $V^{n}$ "on the whole" also is its local geodesic mapping. But there exist wide classes of Riemannian spaces that locally admit non-trivial (different from the affine) geodesic mappings and, at the same time, don't admit such mappings "on the whole" ([5, 6], for example).

In the case when Riemannian space $V^{n}$ doesn't admit non-trivial geodesic mappings, locally or "on the whole", we say that the space is geodesically uniquely defined, correspondingly, locally or "on the whole", in the sense that its object of affine connection is uniquely defined by the totality of its geodesic lines.

In his time T. Levi-Civita has proved (see, for example, [4]) that the problem of does or doesn't the given Riemannian space $V^{n}$ admit locally or "on the whole" some non-trivial geodesic mappings reduces to the problem of existence in some neighborhood of the every point of the space or in the space "on the whole" some twice covariant symmetric, non-singular, smooth of the class $C^{r-1}$ tensor field $\bar{g}$ and gradient, smooth of the class $C^{r-2}$ covariant vector $\psi$, that doesn't equal to zero identically, corresponding components $\bar{g}_{i j}$ and $\psi_{i}$ of which satisfy the differential equations

$$
\bar{g}_{i j, k}=2 \psi_{k} \bar{g}_{i j}+\psi_{i} \bar{g}_{k j}+\psi_{j} \bar{g}_{k i} .
$$

Later N. S. Sinyukov has passed [7] from equations (2) to the equivalent differential equations of the form

$$
a_{i j, k}=\lambda_{i} g_{j k}+\lambda_{j} g_{i k}
$$

according to the components $a_{i j}$ of some symmetric, nonsingular, twice covariant, smooth of the class $C^{r-1}$ tensor $a$ and the components $\lambda_{i}$ of some smooth of the class $C^{r-2}$ gradient covariant vector $\lambda$ that doesn't equal to zero identically, to the so called new form of the basic equations of the theory of geodesic mappings of Riemannian spaces. As a result of analysis the differential prolongations of equation (3) N. S. Sinyukov has received $[7,8]$ a linear system of differential equations of the first order, of Cauchy type, with uniquely defined in the every coordinate neighborhood of the space $V^{n}$ coefficients that, in contrast to equations (2) and generated by them the corresponding system of differential equations, already admits regular methods of investigations.

Equations (3) and received on their base system of linear differential equations characterize both local geodesic mappings and geodesic mappings "on the whole". Their solutions in Riemannian space $V^{n}$ have then, correspondingly, local or global character. By this, it is clear that existence of some solution of equations (2) or (3) even "on the whole" for every coordinate neighborhood of some atlas of the space $V^{n}$ doesn't guarantee the existence of such solution in the space $V^{n}$ "on the whole".

Smooth of the class $C^{r}$ Riemannian space $V^{n}, n, r$ $\in N, n>1, r>1$, with the smooth of the class $C^{r-1}$ metric tensor $g$ is called [4] an equidistant space if in it there exist such smooth of the class $C^{\prime-1}$ covariant vector field $\rho$ and such smooth of the class $C^{r-1}$ invariant $\psi$, that, according to every local coordinate system, the next equalities take place

$$
\rho_{i, j}=\psi g_{i j} .
$$

Here $g_{i j}, \rho_{i}, \psi$ are, correspondingly, coordinate representations of the metric tensor $g$ the covariant vector 
$\rho$ and the invariant $\psi$ according to the considered coordinate neighborhood, “” denotes covariant differentiation according to the determined by the metric tensor $g$ object of affine connection. Equidistant Riemannian space $V^{n}$ is called an equidistant space of general type if the invariant $\psi$ does not identically equals to zero.

For equidistant Riemannian spaces of general type the solutions of the equations (3) are built (see, for example, $[4,8])$. The last one means that locally-equidistant Riemannian spaces locally admit non-trivial geodesic mappings, equidistant Riemannian spaces "on the whole" admit such mappings "on the whole".

\section{Materials and Methods}

In $(n+1)$-dimensional $(n \in N, n \geq 2)$ Euclidean space $E^{n+1}$ with the fixed Cartesian coordinate system $O x^{1} x^{2} \ldots x^{n}$, we will consider sphere $S^{n}$ as a geometrical figure, given by equation

$$
\left(x^{1}\right)^{2}+\left(x^{2}\right)^{2}+\ldots+\left(x^{n}\right)^{2}+\left(x^{n+1}-1\right)^{2}=1 .
$$

It is a unit sphere with the center at the point $\mathrm{Q}(0 ; 0 ; \ldots ; 0 ; 1)$. The space $E^{n+1}$ we will consider to be endowed with the natural topology, the base of which consists of all open $\varepsilon$-balls $(\varepsilon>0)$ with centers in arbitrary points (the sets that are given according to the chosen coordinate system by inequalities

$$
\left.\left(x^{1}-x_{0}^{1}\right)^{2}+\left(x^{2}-x_{0}^{2}\right)^{2}+\ldots+\left(x^{n}-x_{0}^{n}\right)^{2}<\varepsilon^{2}\right),
$$

that is with topology induced by its natural metric. As it is well-known ([9], for example) such topological space is a Hausdorff space and has a countable base. We will consider the sphere $S^{n}$ as a topological subspace of the space. By the hereditary character of properties to be a Hausdorff space and to have a countable base (see, for example, [10]) subspace $S^{n}$ will have the mentioned properties. Also, it is known (see, for example, [11]) that received by such a way topological space $S^{n}$ is compact.

We will turn the topological space $S^{n}$ into the topological manifold by setting on it an atlas that consists of two charts. The so-called stereographic projection will be used for it.

According to (4) sphere $S^{n}$ contains such diametrically opposite points as the origin $O$ of the coordinate system and the point $P(0 ; 0 ; \ldots ; 0 ; 2)$. Let us assume the following: $U_{1}=S^{n} \backslash O ; U_{2}=S^{n} \backslash P$. The both sets are open according to the established on $S^{n}$ topology, because $U_{1}=S^{n} \cap O_{1}, U_{2}=S^{n} \cap O_{2}$, where $O_{1}$ and $O_{2}$ are the open balls of the space $E^{n+1}$, given, correspondingly, by inequalities

$$
\left(x^{1}\right)^{2}+\left(x^{2}\right)^{2}+\ldots+\left(x^{n}\right)^{2}<4,
$$

and

$$
\left(x^{1}\right)^{2}+\left(x^{2}\right)^{2}+\ldots+\left(x^{n}\right)^{2}+\left(x^{n+1}-2\right)^{2}<4 .
$$

It is easy to verify that the mapping $\varphi_{1}: U_{1} \rightarrow R^{n}$, in analytical form given by functions

$$
\varphi_{1}: u^{i}=\frac{x^{i}}{x^{n+1}}, \quad i=\overline{1, n},
$$

is a homeomorphic mapping of the set $U_{1}$, considered as a topological subspace of the space $S^{n}$, onto the space $R^{n}$ endowed with its natural topology. Coordinates of points of the space $R^{n}$ in this case are denoted as $\left(u^{1} ; u^{2} ; \ldots ; u^{n}\right)$. The inverse homeomorphism $\varphi_{1}^{-1}: R^{n} \rightarrow U_{1}$ is given by functions

$$
\varphi_{1}^{-1}:\left\{\begin{array}{l}
x^{i}=\frac{2 u^{i}}{a}, i=\overline{1, n} \\
x^{n+1}=\frac{2}{a},
\end{array}\right.
$$

where $a=1+\left(u^{1}\right)^{2}+\ldots+\left(u^{n}\right)^{2}$. Homeomorphism $\varphi_{1}$ turns the set $U_{1}$ into the coordinate neighborhood with the local coordinates $u^{1} ; u^{2} ; \ldots ; u^{n}$.

By the same way the mapping $\varphi_{2}: U_{2} \rightarrow R^{n}$, in analytical form given by functions

$$
\varphi_{2}: v^{i}=\frac{x^{i}}{2-x^{n+1}}, \quad i=\overline{1, n},
$$

is a homeomorphic mapping of the set $U_{2}$, considered as a topological subspace of the space $S^{n}$, onto the space $R^{n}$ endowed with its natural topology. Coordinates of points of the space $R^{n}$ this time are denoted as $\left(v^{1} ; v^{2} ; \ldots ; v^{n}\right)$. The inverse homeomorphism $\varphi_{2}{ }^{-1}$ : $R^{n} \rightarrow U_{2}$, is given by functions

$$
\varphi_{2}^{-1}:\left\{\begin{array}{l}
x^{i}=\frac{2 v^{i}}{b}, i=\overline{1, n} \\
x^{n+1}=\frac{2(b-1)}{b},
\end{array}\right.
$$

where $b=1+\left(v^{1}\right)^{2}+\left(v^{2}\right)^{2}+\cdots+\left(v^{n}\right)^{2}$. Homeomor-phism $\varphi_{2}$ turns the set $U_{2}$ into coordinate neighborhood with the local coordinates $v^{1} ; v^{2} ; \ldots ; v^{n}$.

Finally, the space $S^{n}$ has turned out to be covered by coordinate neighborhoods $U_{1}$ and $U_{2}$ of two charts $\left(U_{1} ; \varphi_{1}\right)$ and $\left(U_{2} ; \varphi_{2}\right) . U_{1} \cap U_{2}=S^{n} \backslash\{O ; P\}$. For $U_{1} \cap$ $U_{2}$ functions of transformation of coordinates $u^{i}$ to coordinates $v^{i}$ and the inverse ones represent transformation of the set $R^{n} \backslash(0 ; 0 ; \ldots ; 0)$ and are of the form:

$$
\begin{aligned}
& \varphi_{2} \circ \varphi_{1}^{-1}: v^{i}=\frac{u^{i}}{a-1}, i=\overline{1, n} ; \\
& \varphi_{1} \circ \varphi_{2}^{-1}: u^{i}=\frac{v^{i}}{b-1}, i=\overline{1, n} .
\end{aligned}
$$

Thus, they are smooth functions of the class $C^{\infty}$ on their domains. From the geometrical point of view functions (7) establish inversion of the space $R^{n}$ with respect to a unit sphere $S^{n-1}$ with the center at the point $(0 ; 0 ; \ldots ; 0)$.

By such a way the sphere $S^{n}$ is turned to the $n$-dimensional real differentiable $C^{\infty}$-manifold with the atlas that consists of two charts, hypersurface, submanifold of Euclidean space $E^{n+1}$ with natural atlas of one chart, determined by the fixed in $E^{n+1}$ Cartesian coordinate system.

We will turn the manifold $S^{n}$ to Riemannian subspace of the space $E^{n+1}$ by traditional for Riemannian geometry way, introducing the components $g_{i j} ; i, j=\overline{1, \mathrm{n}}$, of the corresponding metric tensor $g$ for the every of its charts as 
following.

Let some point $M\left(x^{1} ; x^{2} ; \ldots ; x^{n+1}\right)$ of the sphere $S^{n}$ belongs to the coordinate neighborhood $U_{1}$. Coordinates of radius-vector $\overrightarrow{\mathrm{r}}=\overrightarrow{O M}$ of the point, as the functions of parameters $u^{1} ; u^{2} ; \ldots ; u^{n}$, are the next

$$
\vec{r}\left(\frac{2 u^{1}}{a} ; \frac{2 u^{2}}{a} ; \ldots ; \frac{2 u^{n}}{a} ; \frac{2}{a}\right) .
$$

Then

$$
\begin{gathered}
\vec{r}_{l}=\frac{\partial \vec{r}}{\partial u^{i}}=\left(-\frac{4 u^{1} u^{i}}{a^{2}} ;-\frac{4 u^{2} u^{i}}{a^{2}} ; \ldots ; \frac{2}{a}-\right. \\
\left.-\frac{4\left(u^{i}\right)^{2}}{a^{2}} ; \ldots ;-\frac{4 u^{n} u^{i}}{a^{2}} ;-\frac{4 u^{i}}{a^{2}}\right), \\
g_{i j}=\vec{r}_{l} \cdot \vec{r}_{J} \quad \text { and, by this, } \\
\left\{\begin{array}{l}
g_{i i}=\frac{4}{a^{2}}, i=\overline{1, n}, \\
g_{i j}=0 ; i \neq j ; i, j=\overline{1, n}
\end{array}\right.
\end{gathered}
$$

It is easy to calculate that the elements $g^{i j}$ of the matrix, inverse to the matrix $\left\|g_{i j}\right\|, \quad i, j=\overline{1, n}$ in this case are the next

$$
\left\{\begin{array}{l}
g^{i i}=\frac{a^{2}}{4}, \quad i=\overline{1, n} \\
g^{i j}=0 ; \quad i \neq j ; \quad i, j=\overline{1, n} .
\end{array}\right.
$$

By the analogy to this, according to the coordinate neighborhood $U_{2}$, we have

$$
\begin{aligned}
& \left\{\begin{array}{l}
g_{i i}=\frac{4}{b^{2}}, \quad i=\overline{1, n}, \\
g_{i j}=0 ; \quad i \neq j ; \quad i, j=\overline{1, n} .
\end{array}\right. \\
& \begin{cases}g^{i i}=\frac{b^{2}}{4}, \quad i=\overline{1, n}, \\
g^{i j}=0 ; \quad i \neq j ; \quad i, j=\overline{1, n} .\end{cases}
\end{aligned}
$$

Formulas (8) and (10) indicate that the metric tensor $g$ defines on $S^{n}$ a positive definite quadratic form - for the matrixes $\left\|g_{i j}\right\|$, built of its components according to the both charts, conditions of Silvester's criteria of positive definiteness of quadratic forms ([12], for example) are evidently satisfied.

Formulas (8)-(11) admit to find in explicit form for the each of the charts $\left(U_{1} ; \varphi_{1}\right)$ and $\left(U_{2} ; \varphi_{2}\right)$ components $\Gamma_{i j}^{h}$ of the object of affine connection of the received Riemannian space.

As it is known ([1], for example), in general case

$$
\begin{gathered}
\Gamma_{i j}^{h}\left(t^{1}, \ldots, t^{n}\right)=\frac{1}{2} g^{h \alpha}\left(\frac{\partial g_{i \alpha}}{\partial t^{j}}+\frac{\partial g_{j \alpha}}{\partial t^{i}}-\frac{\partial g_{i j}}{\partial t^{\alpha}}\right), \\
h, i, j, \alpha=\overline{1, n}
\end{gathered}
$$

By (8) and (9) in the chart $\left(U_{1} ; \varphi_{1}\right)$ we have:

$$
\Gamma_{i i}^{i}=-\frac{2 u^{i}}{a} ; \quad i=\overline{1, n} ;
$$

(there is no summing by the index $i$ )

$$
\Gamma_{i i}^{h}=\frac{2 u^{h}}{a} ; \quad h, i=\overline{1, n} ;
$$

(there is no summing by the index $i$ )

$$
\Gamma_{i j}^{i}=\Gamma_{j i}^{i}=-\frac{2 u^{i}}{a} ; i, j=\overline{1, n} ;
$$

(there is no summing by the index $i$ )

$$
\Gamma_{i j}^{h}=0, \quad i \neq h, \quad j \neq h, \quad i \neq j, \quad h, i, j=\overline{1, n} .
$$

By the analogy, in the chart $\left(U_{2} ; \varphi_{2}\right)$, according to (10) and (11) we have

$$
\Gamma_{i i}^{i}=-\frac{2 v^{i}}{b} ; \quad i=\overline{1, n} ;
$$

(there is no summing by to the index $i$ )

$$
\Gamma_{i i}^{h}=\frac{2 v^{h}}{a} ; \quad h, i=\overline{1, n} ;
$$

(there is no summing by the index $i$ )

$$
\Gamma_{i j}^{i}=\Gamma_{j i}^{i}=-\frac{2 v^{i}}{b} ; i, j=\overline{1, n} ;
$$

(there is no summing by the index $i$ )

$$
\Gamma_{i j}^{h}=0, \quad i \neq h, \quad j \neq h, \quad i \neq j, \quad h, i, j=\overline{1, n} .
$$

In [13] Ishihara has proved that the sphere $S^{n}$ - the built Riemannian space $S^{n}-$ is an equidistant Riemannian space. As we can see, it is an equidistant Riemannian space "on the whole". Indeed, the function

$$
\rho=1-x^{\mathrm{n}+1}
$$

is defined at every point of the sphere $S^{n}$ that is given by equation (4) according to the chosen in $E^{n+1}$ Cartesian coordinate system. Coordinate representation of the function $\rho$ up to the chart $\left(U_{1} ; \varphi_{1}\right)$ looks like

$$
\rho_{1}=\frac{a-2}{a}, \quad a=\left(u^{1}\right)^{2}+\left(u^{2}\right)^{2}+\ldots+\left(u^{n}\right)^{2}+1
$$

And up to the chart $\left(U_{2} ; \varphi_{2}\right)$ coordinate representation of this function has the form

$$
\rho_{2}=\frac{2-b}{b}, \quad b=\left(v^{1}\right)^{2}+\left(v^{2}\right)^{2}+\ldots+\left(v^{n}\right)^{2}+1
$$

Thus, according to the chart $\left(U_{1} ; \varphi_{1}\right)$ we have

$$
\begin{gathered}
\rho_{i}=\frac{1}{\partial u^{i}}=\frac{4 u^{i}}{a^{2}}, \\
\rho_{i, i}=\frac{\partial \rho_{i}}{\partial u^{i}}-\rho_{\alpha} \Gamma_{i i}^{\alpha}=-\frac{4(a-2)}{a^{3}}
\end{gathered}
$$

(there is no summing by the index $i$ )

$$
\rho_{i, j}=\frac{\partial \rho_{i}}{\partial u^{j}}-\rho_{\alpha} \Gamma_{i j}^{\alpha}=0, \quad i \neq j, \quad i, j=\overline{1, n} .
$$

It follows from this that, according to the chart $\left(U_{1} ; \varphi_{1}\right)$,

$$
\begin{gathered}
\rho_{i, j}={ }_{1} g_{i j} \\
1
\end{gathered} \text {, where } \begin{aligned}
& \psi \\
& 1
\end{aligned}=\frac{2-a}{a}, i, j=\overline{1, n} .
$$

By the analogy, up to the chart $\left(U_{2} ; \varphi_{2}\right)$

$$
\rho_{i}=\frac{\partial \rho}{\partial v^{i}}=-\frac{4 v^{i}}{b^{2}}
$$




$$
\underset{2}{\rho_{i, i}}=\frac{\partial \rho_{i}}{\partial v^{i}}-\underset{2}{\rho_{\alpha}} \Gamma_{i i}^{\alpha}=-\frac{4(b-2)}{b^{3}},
$$

(there is no summing by the index $i$ )

$$
\rho_{i, j}=\frac{\partial \rho_{i}}{\partial v^{j}}-\underset{2}{\rho_{\alpha}} \Gamma_{i j}^{\alpha}=0, \quad i \neq j, \quad i, j=\overline{1, n} .
$$

So, up to the chart $\left(U_{2} ; \varphi_{2}\right)$ we also have

$$
\rho_{i, j}=\frac{\psi g_{i j}}{2}, \text { where } \frac{\psi}{2}=\frac{b-2}{b}, i, j=\overline{1, n} .
$$

At the same time, it is clear that functions $\frac{\psi}{1}$ and $\frac{\psi}{2}$ are the coordinate representations, correspondingly, according to the charts $\left(U_{1} ; \varphi_{1}\right)$ and $\left(U_{2} ; \varphi_{2}\right)$ of the function $\psi=-\rho$, that is defined on $S^{n}$ "on the whole".

Thus, the considered sphere $S^{n}$ is "on the whole" an equidistant Riemannian space with the gradient concircular vector field that is defined in the chart $\left(U_{1} ; \varphi_{1}\right)$ by the law (12), and in the chart $\left(U_{2} ; \varphi_{2}\right),-$ by the law (13).

The function $\psi=1-x^{n+1}$ equals to zero only on the sphere $S^{n-1} \subset S^{n}$ that in the space $E^{n+1}$ is given by the system of equations

$$
\left\{\begin{array}{l}
\sum_{i=1}^{n}\left(x^{i}\right)^{2}=1 \\
x^{n+1}=1
\end{array}\right.
$$

This means that $\psi$ isn't equal to zero on $S^{n}$ identically, the considered space $S^{n}$ is an equidistant Riemannian space of general type.

As it is known ([5,8]), in an equidistant Riemannian space $V^{n}$ tensor $a$ and vector $\lambda$, components of which according to the corresponding coordinate neighborhood are of the form

$$
\begin{gathered}
a_{i j}=c \rho_{i} \rho_{j}+c_{1} g_{i j}, \\
\lambda_{i}=c \psi \rho_{i},
\end{gathered}
$$

where " $c$ " and " $c_{1}$ " are some constants, generate, correspondingly locally or "on the whole", some non-trivial solution of equations (3) if $a$ and $\lambda$ have the necessary class of smooth, $\lambda$ isn't equal to zero identically, matrix $\left\|a_{i j}\right\|$ is non-singuar in the corresponding coordinate neighborhoods. By this, it is obvious, that the restriction $\mathrm{c} \neq 0$ is urgent.

In our case, for the sphere $S^{n}$, invariant $\psi$ identically isn't equal to zero, $\rho_{i}$ are the components of vector-gradient of the function $\rho=1-x^{n+1}$. As we have shown by (12) and (13), $\rho_{i}$ identically are not equal to zero. It follows from this, that $\lambda_{i}$ identically are not equal to zero on the sphere $S^{n}$ Also it is obvious, that covariant vector $\lambda_{i}$ is a vector-gradient for the function $\Lambda=-\frac{c}{2} \psi^{2}$.

We may obtain non-singular matrix $\left\|a_{i j}\right\|$ in some neighborhood of the every point of $S^{n}$ by choosing the constant $c_{1}$, because $\operatorname{det}\left\|g_{i j}\right\| \neq 0$ everywhere on $S^{n}$.
Let's show that such constant $c_{1}$ may be chosen for $S^{n}$ "on the whole".

As $c=$ const,$\quad c \neq 0$, from (14) we have

$$
\frac{1}{c} a_{i j}=\rho_{i} \rho_{j}-q g_{i j} \text {, }
$$

where

$$
q=-\frac{c_{1}}{c}
$$

So, the task is reduced to the corresponding choice of the constant $q$. It is necessary to prove that it can be chosen in such a way that at the every point of $S^{n}$, according to any corresponding chart,

$$
\operatorname{det}|| \rho_{i} \rho_{j}-q g_{i j} \| \neq 0
$$

In our case, obviously, we have to consider an arbitrary point of the two chosen on $S^{n}$ charts.

According to the chart $\left(U_{1} ; \varphi_{1}\right)$, as well as to the chart $\left(U_{2} ; \varphi_{2}\right)$, we can consider an equation

$$
\operatorname{det}|| \rho_{i} \rho_{j}-q g_{i j} \|=0
$$

It is a characteristic equation of the following pencil $\rho_{i} \rho_{j} \xi^{i} \xi^{j}-q g_{i j} \xi^{i} \xi^{j}$ of forms, of a regular pencil of forms because the form $g_{i j} \xi^{i} \xi^{j}$ is positive-defined.

It is known (see [12], for example), that the equation (16) has exactly $n$ real roots $q_{1}, q_{2}, \ldots, q_{n}$ and

$$
q_{\text {min }}=\min _{\sum_{i=1}^{n}\left(\xi^{i}\right)^{2}>0} \frac{\rho_{i} \rho_{j} \xi^{i} \xi^{j}}{g_{\alpha \beta} \xi^{\alpha} \xi^{\beta}}
$$

Let's consider the last one relation separately according to the given two charts. By (5), (8), (12) in the chart $\left(U_{1} ; \varphi_{1}\right)$, we have

$$
\frac{\frac{\rho_{i} \rho_{j} \xi^{i} \xi^{j}}{g_{\alpha \beta} \xi^{\alpha} \xi^{\beta}}}{g^{\prime}}=\frac{\sum_{i, j=1}^{n} x^{i} x^{j} \xi^{i} \xi^{j}}{\sum_{i=1}^{n}\left(\xi^{i}\right)^{2}}
$$

By (6), (9), (13) the analogous relations are true for the chart $\left(U_{2} ; \varphi_{2}\right)$.

From the other hand,

$$
\begin{aligned}
& \min _{\sum_{i=1}^{n}\left(\xi^{i}\right)^{2}>0} \frac{\sum_{i, j=1}^{n} x^{i} x^{j} \xi^{i} \xi^{j}}{\sum_{i=1}^{n}\left(\xi^{i}\right)^{2}}= \\
& =\min _{\substack{i=1 \\
\sum_{=1}\left(\xi^{i}\right)^{2}>0}} \sum_{i, j=1}^{n} x^{i} x^{j} \cdot \frac{\xi^{i}}{\sqrt{\sum_{k=1}^{n}\left(\xi^{k}\right)^{2}}} \cdot \frac{\xi^{j}}{\sqrt{\sum_{k=1}^{n}\left(\xi^{k}\right)^{2}}}= \\
& =\min _{\substack{n=1 \\
\sum_{i=1}\left(\eta_{i}\right)^{2}=1}} \sum_{i=1}^{n} x^{i} x^{j} \eta^{i} \eta^{j}
\end{aligned}
$$

where

$$
\eta^{i}=\frac{\xi^{i}}{\sqrt{\sum_{k=1}^{n}\left(\xi^{k}\right)^{2}}}, \quad i=\overline{1, n} .
$$

Thus, we speak about minimization of the continuous function 


$$
q\left(x^{1}, \ldots, x^{n}, x^{n+1} ; \eta^{1}, \eta^{2}, \ldots, \eta^{n}\right)=\sum_{i=1}^{n} x^{i} x^{j} \eta^{i} \eta^{j} .
$$

with the domain $S^{n} \times S^{n-1}$

$$
\left(\mathrm{x}^{1}, \ldots, \mathrm{x}^{\mathrm{n}}, \mathrm{x}^{\mathrm{n}+1}\right) \in S^{n} ;\left(\eta^{1}, \eta^{2}, \ldots, \eta^{\mathrm{n}}\right) \in \mathrm{S}^{\mathrm{n}-1} .
$$

The set $S^{n} \times S^{n-1}$ is compact (see, for example, [11]), every continuous function reaches on it its minimal and maximal values. Denote the minimal value of our function as $q^{*}$. Then, in every point $M\left(x^{1}, \ldots, x^{n}, x^{n+1}\right) \quad £^{n}$, in every chart $\left(U_{1} ; \varphi_{1}\right), \mathrm{i}=1,2$, it is true that $q^{*} \leq q_{\text {min }}$. Thus, if as the number $q\left(=-\frac{c_{1}}{c}\right)$ we will take any real number less then $q^{*}$, the condition (15) will take place in every point of $S^{n}$.

Thus, on the ground of the sphere $S^{n}$ that is considered as a subspace of Euclidean space $E^{n+1}, n \in N$, $n \geq 1$, an example of the global solution of the new form of the basic equations of the theory of geodesic mappings of Riemannian space is built.

\section{Results and Discussion}

In [13] Ishihara $S$. have proved that sphere $S^{n}$, considered as a subspace of Euclidean space $E^{n+1}(n \in N)$, is an equidistant Riemannian space "on the whole". The result opened the realized in the present paper theoretical opportunities for building on the ground of a sphere the global non-trivial solution of equations (3) for compact Riemannian space. In [14] Mike`s J. has built for the sphere $S^{n}$ an example of non-trivial solution of equations (2). By such a way it has been proved that sphere admit non-trivial geodesic mappings "on the whole". But the problem of application of equations (3) for finding examples of non-trivial geodesic mappings of Riemannian spaces "on the whole" in the theory of such mappings remains actual. It is so especially by the fact that we can state ([8], for example) that the overwhelming majority of Riemannian spaces don't admit such mappings even in the local form. In particular, it seems to be interesting to investigate basing on the results of the work [15] a sphere in Galilean space of different dimensions according to its ability to admit non-trivial geodesic mappings and geodesic transformations as locally, as on the whole.

\section{Conclusions}

An example of the global solution to the so-called new form of basic equations of the theory of geodesic mappings of Riemannian spaces is built in the article. It seems that methods that have been used may be spread to the more wide classes of spaces.

\section{REFERENCES}

[1] Sinyukov N. S., "Preliminary information," in Geodesic mappings of Riemannian spaces, 1st ed, Nauka, Moskow, 1979, pp. 11-69

[2] Kobayashi S, Nomizu K, "Riemannian Connections," in Foundations of Differential Geometry, vol. I, 1st ed, Interscience, New York/London, 1963, pp.154-197

[3] Kagan V. F., "General Foundations of Surface Theory", in Foundations of the theory of surfaces. Part 1, 1st ed, OGIZ Gostechiz, Moskow, Leningrad, 1947, pp. 195-252

[4] Sinyukov N. S., "Foundations of the theory of geodesic mappings of Riemannian spaces," in Geodesic mappings of Riemannian spaces, 1st ed, Nauka, Moskow, 1979, pp. $70-119$

[5] Sinyukov N. S., "Basic theorems of the theory of geodesic mappings of Riemannian spaces," in Geodesic mappings of Riemannian spaces, 1st ed, Nauka, Moskow, 1979, pp. 120-155

[6] Mike`s J., Stepanov S. E., Differential geometry of special mappings, 1st ed, Palacky Univ. Press, Olomouc, 2015.

[7] Sinyukov N. S., Onto the theory of geodesic mappings of Riemannian spaces, Docl. Akad. Nauk SSSR, Vol.169, No.4, 770-772, 1966.

[8] Mike`s J., Van`zurova A., J. Hinterleitner, "Mappings between Riemannian manifolds," in Geodesic Mappings and Some Generalizations, 1st ed, Palacky Univ. Press, Olomouc, 2009, pp. 167-184

[9] Engelking R., "Metric and metrizable spaces," in General Topology, 1st ed, Polish Scientific publishers, Warszawa, 1977, pp. 310-372

[10] Engelking R., "Operations n topological spaces," in General Topology, 1st ed, Polish Scientific publishers, Warszawa, 1977, pp. 93-164

[11] Engelking R., "Compact spaces," in General Topology, Polish Scientific publishers, 1st ed, Warszawa, 1977, pp. 165-309

[12] Gantmaher F. B., "Quadratic and Hermitian forms," in Theory of matrixes, 1st ed, Gostexiz, Moskow, 1953, pp. 267-310.

[13] Ishihara S., On infinitesimal consircular transformations, Kodai Math. Sem. Rep., Vol.12, No. 2, 45-56, 1960.

[14] Mikes $s$ J., On the existence of $n$-dimensional compact Riemannian spaces admitting nontrivial global projective transformations, Docl. Akad. Nauk SSSR, Vol.305, 534-536, 1989.

[15] Artykbaev Abdullaaziz, Nurbayev Abdurashid Ravshanovich, "The Indicatrix of the Surface in Four-Dimensional Galilean Space," Mathematics and Statistics, Vol. 8, No. 3, pp. 306 - 310, 2020. DOI: 10.13189/ms.2020.080309. 